\title{
Effects of Topical Retinoids on Acne and Post-inflammatory Hyperpigmentation in Patients with Skin of Color: A Clinical Review and Implications for Practice
}

\author{
Valerie D. Callender ${ }^{1,2}\left(\right.$ D Hilary Baldwin ${ }^{3,4} \cdot$ Fran E. Cook-Bolden $^{5,6} \cdot$ Andrew F. Alexis $^{6} \cdot$ Linda Stein Gold $^{7}$. \\ Eric Guenin ${ }^{8}$
}

Accepted: 28 September 2021 / Published online: 9 November 2021

(c) The Author(s) 2021

\begin{abstract}
Acne is a common cause for post-inflammatory hyperpigmentation (PIH), particularly in patients with skin of color (SOC), and PIH is often more distressing to patients than the acne itself. Topical retinoids are approved for the treatment of acne and for pigmentation disorders such as melasma or mottled hyperpigmentation associated with photodamage; moreover, they have been shown to reduce hyperpigmentation in patients with SOC. Therefore, treatment with topical retinoids should be started as early as possible unless contraindicated. Use of novel formulations or application of commonly recommended moisturizers may help reduce irritation. Combining retinoids with other topical agents and procedures such as superficial chemical peels can help to improve hyperpigmentation. Primary acne lesions are likely to improve weeks before PIH resolves and helping patients manage their expectations may reduce frustration. Providing clinicians and researchers with more education about the presentation and management of dermatologic conditions in patients with SOC is also recommended.
\end{abstract}

Ortho Dermatologics is a division of Bausch Health, US, LLC.

Valerie D. Callender

drcallender@callenderskin.com

1 Callender Dermatology and Cosmetic Center, 12200 Annapolis Road, Suite 315, Glenn Dale, MD 20769, USA

2 Department of Dermatology, Howard University College of Medicine, Washington, DC, USA

3 The Acne Treatment and Research Center, Brooklyn, NY, USA

4 Robert Wood Johnson University Hospital, New Brunswick, NJ, USA

5 Fran E. Cook-Bolden, MD, PLLC, New York, NY, USA

6 Weill Cornell Medical College, New York, NY, USA

7 Henry Ford Hospital, Detroit, MI, USA

8 Ortho Dermatologics, Bridgewater, NJ, USA

\section{Key Points}

For patients with skin of color (SOC), hyperpigmentation due to acne may be more distressing than the acne itself.

Rapid effective treatment of acne is an important strategy for improving post-inflammatory hyperpigmentation (PIH) due to acne.

Evidence from clinical trials indicate that topical retinoids should be considered a first-line therapy in patients with acne and hyperpigmentation, unless contraindicated (e.g., pregnancy); for patients with sensitive skin, retinoids with new vehicle formulations may be less irritating than older formulations.

Education and training for clinicians and researchers about acne and PIH in patients with SOC may improve diagnoses and treatment outcomes; education for patients about their dermatologic conditions, medications, and skin care may help to manage expectations and improve treatment adherence. 


\section{Introduction}

Hyperpigmentation is one of the most common reasons for individuals with skin of color (SOC) to seek dermatologic care [1]. Post-inflammatory hyperpigmentation (PIH), which includes acne-induced hyperpigmentation, occurs when inflammation or injury results in overproduction of melanin or abnormal deposition of melanin in the epidermis or dermis $[2,3]$. PIH lesions in the epidermis, which are generally tan to dark brown, can persist for up to 12 months $[1,3,4]$. Dermal lesions tend to have a blue/gray color and can persist for years.

PIH can be caused by a number of different cutaneous injuries, such as insect bites, topical irritants, burns, and papulosquamous disorders [5]. However, acne is one of the major causes of PIH, particularly in patients with melaninrich skin $[3,5]$. These patients can have hyperpigmented acne lesions, hyperpigmented macules, or both (Fig. 1). In a 2002 study of acne in patients with SOC, hyperpigmented macules were found in $65.3 \%, 52.7 \%$, and $47.4 \%$ of Black, Hispanic, and Asian participants, respectively [6]. Moreover, as indicated by a study from the National Skin Centre in Singapore, PIH is more common among racial/ethnic groups with higher skin phototypes [7]. People with SOC comprise a majority of the world's population and are the fastest-growing segment of the US population [1]. Given this current demographic landscape, it is important to discuss best practices for assessing and treating dermatologic conditions such as PIH.

Hyperpigmentation may be more distressing than acne in patients with SOC; moreover, patients with higher skin phototypes may find hyperpigmentation more distressing than patients with lower skin phototypes [2]. Studies of acne in female patients have reported racial/ethnic differences as follows: hyperpigmentation was more prevalent among Black women (65\%) than Hispanic (48\%), White (25\%), Asian (18\%), or Indian (10\%) women [8]; PIH was more bothersome to Black women (18.8\%) than Hispanic (10.9\%), Asian/other (7.4\%), or White (2.8\%) women [9]; and concern about clearing $\mathrm{PIH}$ was more common in nonWhite women (41.6\%) than White women (8.4\%) [10].

Since hyperpigmentation is an acne sequela, early and aggressive acne treatment is important for the management and prevention of PIH in patients with SOC [2]. However, this approach must be balanced against the risk of treatmentrelated skin irritation, with resultant dyspigmentation of its own and worsening of pre-existing hyperpigmentation. Strong consideration should be given to treatments that are less irritating [11] and are effective against both acne and hyperpigmentation.

To address some of the challenges of managing both acne and hyperpigmentation in patients with SOC, we
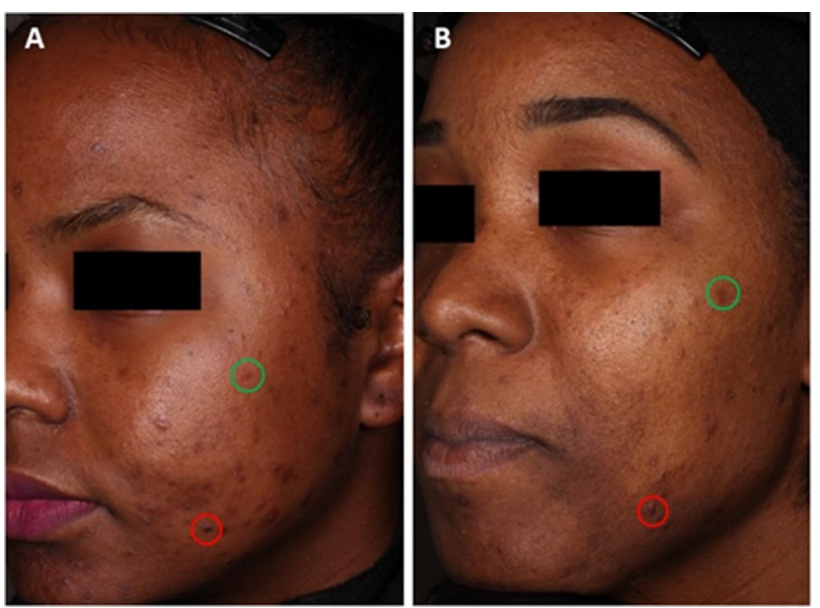

Fig. 1 Hyperpigmented acne lesions (red circles) and hyperpigmented macules (green ovals) in two 27-year-old Black females (A and B) who participated in a phase III study of tazarotene $0.045 \%$ lotion.

conducted a qualitative review of topical retinoids that are currently approved by the US Food and Drug Administration (FDA) for the treatment of acne. Whenever possible, we tried adhering to similar terminology as used in the source materials, such as 'acne-induced hyperpigmentation' when hyperpigmentation was specifically attributed to acne, 'PIH' when hyperpigmentation was specifically described as postinflammatory (due to acne or other cause), and 'hyperpigmentation' when reported as a safety outcome in an acne clinical trial or when a general term was needed. The qualitative review was intended to provide some context for the clinical implications and practical treatment strategies presented in this report, including the importance of effectively treating underlying acne in patients with hyperpigmentation.

\section{Retinoids, Acne, and Hyperpigmentation}

\subsection{Retinoids for the Treatment of Acne}

Topical retinoids are recommended by the American Academy of Dermatology (AAD) for the treatment and management of acne, based on strong levels of evidence from well controlled clinical trials [12]. Retinoids currently approved by the US FDA for the treatment of acne are as follows: tretinoin ( 7 gel doses [0.01\% to $0.1 \%], 3$ cream doses $[0.025 \%$ to $0.1 \%$ ], $0.05 \%$ lotion); adapalene $(0.1 \%$ solution, $0.1 \%$ and $0.3 \%$ gel, $0.1 \%$ cream, $0.1 \%$ lotion); tazarotene $(0.1 \%$ gel, $0.1 \%$ cream, $0.1 \%$ foam, $0.045 \%$ lotion); and trifarotene $(0.005 \%$ cream $)$. These retinoids can be used as monotherapy or in combination with other non-retinoid treatments (see Sect. 4.3) [12]. 
Retinoids exert their biological effects by binding to intranuclear retinoid acid receptors (RAR) or retinoid $\mathrm{X}$ receptors (RXR) [13]. There are three types of RAR receptors $(\alpha, \beta$, and $\gamma$ ) and every retinoid medication has its own distinctive binding properties. The anti-acne effects of retinoids are due to their ability to reduce keratinocyte proliferation and normalize follicular differentiation, which restores normal desquamation and helps to unclog pores [13, 14]. Topical retinoids also have anti-inflammatory effects that include the inhibition of bacterial-induced proinflammatory pathways, suppression of Toll-like receptors, and reduction in the release of cytokines and nitric oxide. Moreover, retinoids can correct pigmentary problems by inhibiting the transfer of melanosomes to keratinocytes and increasing the rate of epidermal turnover [14].

\subsection{Retinoid Effects on Acne and Hyperpigmentation: Older Therapies}

Topical retinoids are a favorable option in patients with SOC because of their proven efficacy in acne and their potential to lighten hyperpigmented lesions. The effects of retinoids on hyperpigmentation have been evaluated in various studies, either as an efficacy or safety assessment, with generally favorable results (Table 1) [15-26].

Tretinoin was the first retinoid specifically evaluated for the treatment of hyperpigmentation in patients with melanin-rich skin. In a 40-week, double-blind, vehicle-controlled study that included 68 Black men and women with hyperpigmented lesions (due to acne, shaving irritation, eczema, ingrown hairs, and folliculitis), improvements were found with tretinoin $0.1 \%$ cream as early as week 4 [26]. However, as expected with older tretinoin formulations, $50 \%$ of active-treated participants experienced cutaneous adverse reactions; these were generally well managed with emollients or temporary medication reduction/discontinuation.

Tazarotene is a selective $\mathrm{RAR}_{\beta} / \mathrm{RAR} \gamma$ agonist whose active form (tazarotenic acid) regulates gene expression by binding to promoter regions (i.e., direct regulation) and to nuclear transcription factors such as AP1, which is associated with cell proliferation and inflammation [27]. Downregulation of cell proliferation and inflammation is known to have lightening effects in skin [28]. Tazarotene $0.1 \%$ cream is FDA-approved for adjunctive use in the mitigation of fine wrinkling, mottled hyper- and hypopigmentation, and benign lentigines in the facial area [29]. Tazarotene $0.1 \%$ cream was also evaluated in an 18 -week, double-blind, vehicle-controlled trial that included 74 patients aged $\geq 12$ years with Fitzpatrick skin types III-VI and acne-induced PIH [23]. In this study, which was specifically designed to evaluate changes in PIH (and not acne), statistically significant reductions were found with tazarotene versus vehicle $(p<0.05)$ for overall hyperpigmentation severity, intensity of hyperpigmented lesions, and area of hyperpigmented lesions. Trace levels of erythema, burning, and peeling were found in both treatment arms; dryness was generally mild.

Tazarotene $0.1 \%$ cream was compared with adapalene $0.3 \%$ gel in a 16 -week, investigator-blinded study that included patients aged $\geq 12$ years with moderate-to-severe acne and a subgroup of 180 patients with clinically detectable PIH at baseline [21]. Although an earlier open-label trial of adapalene $0.1 \%$ gel had shown significant acne lesion reductions and promising PIH results in 44 Black South Africans with moderate acne [25], this investigator-blinded study indicated significantly better acne and PIH outcomes with tazarotene $0.1 \%$ cream compared with adapalene $0.3 \%$ gel. Among non-White participants, PIH index scores (which incorporated severity and distribution of hyperpigmented lesions) significantly decreased from baseline to week 16 for tazarotene $0.1 \%$ cream but not adapalene $0.3 \%$ gel. In addition, mean reductions in PIH index scores were significantly greater with tazarotene versus adapalene $(-48.9 \%$ vs $-4.8 \% ; p=0.017)$.

Studies of combination therapies include a vehicle-controlled study of clindamycin $1.2 \%$ /tretinoin $0.025 \%$ for acne and hyperpigmentation $(N=33)$ [20] and two open-label studies of hydroquinone $4 \% /$ retinol $0.15 \%$ for hyperpigmentation/melasma $(N=21$ and $N=28)$ [22,24]. These studies showed improvements in overall hyperpigmentation severity [20, 22, 24], color/intensity of hyperpigmented lesions [22, 24], lesion counts [22], lesion size [22], lesion area [24], colorimetric measurements [22, 24], and patient-reported global severity [20].

\subsection{Retinoid Effects on Acne and Hyperpigmentation: Newer Formulations and Therapies}

Although retinoids offer benefits in terms of effective acne treatment and favorable hyperpigmentation outcomes, they can be irritating - which in itself can contribute to unwanted pigmentary changes [30]. To address issues of irritation and tolerability, novel lotion formulations were developed for tretinoin and tazarotene [31]. Tretinoin $0.05 \%$ lotion was developed using a polymeric emulsion technology resulting in a honeycomb matrix that provides uniform distribution of the active ingredient (which was micronized for more homogeneous dispersion and to promote diffusion into skin follicular openings), along with a homogeneous dispersion of the moisturizing/hydrating ingredients [32]. While similar to the tretinoin $0.05 \%$ lotion, tazarotene $0.045 \%$ lotion was developed using parent emulsion technology in which the active and moisturizing/hydrating ingredients are encapsulated in oil droplets and uniformly suspended within a threedimensional mesh matrix, which instantly breaks apart upon contact with the skin [33]. These lotion formulations may 


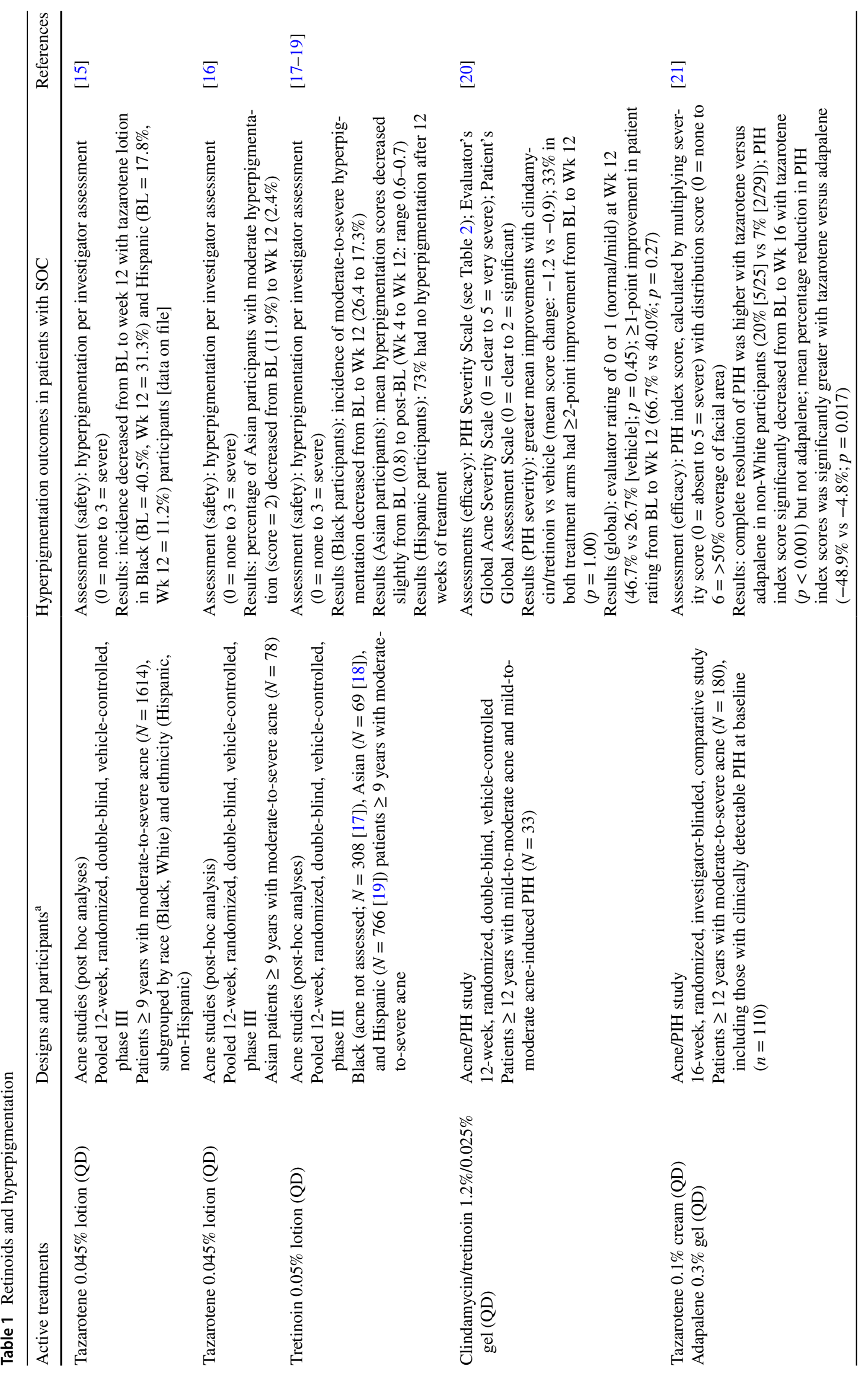




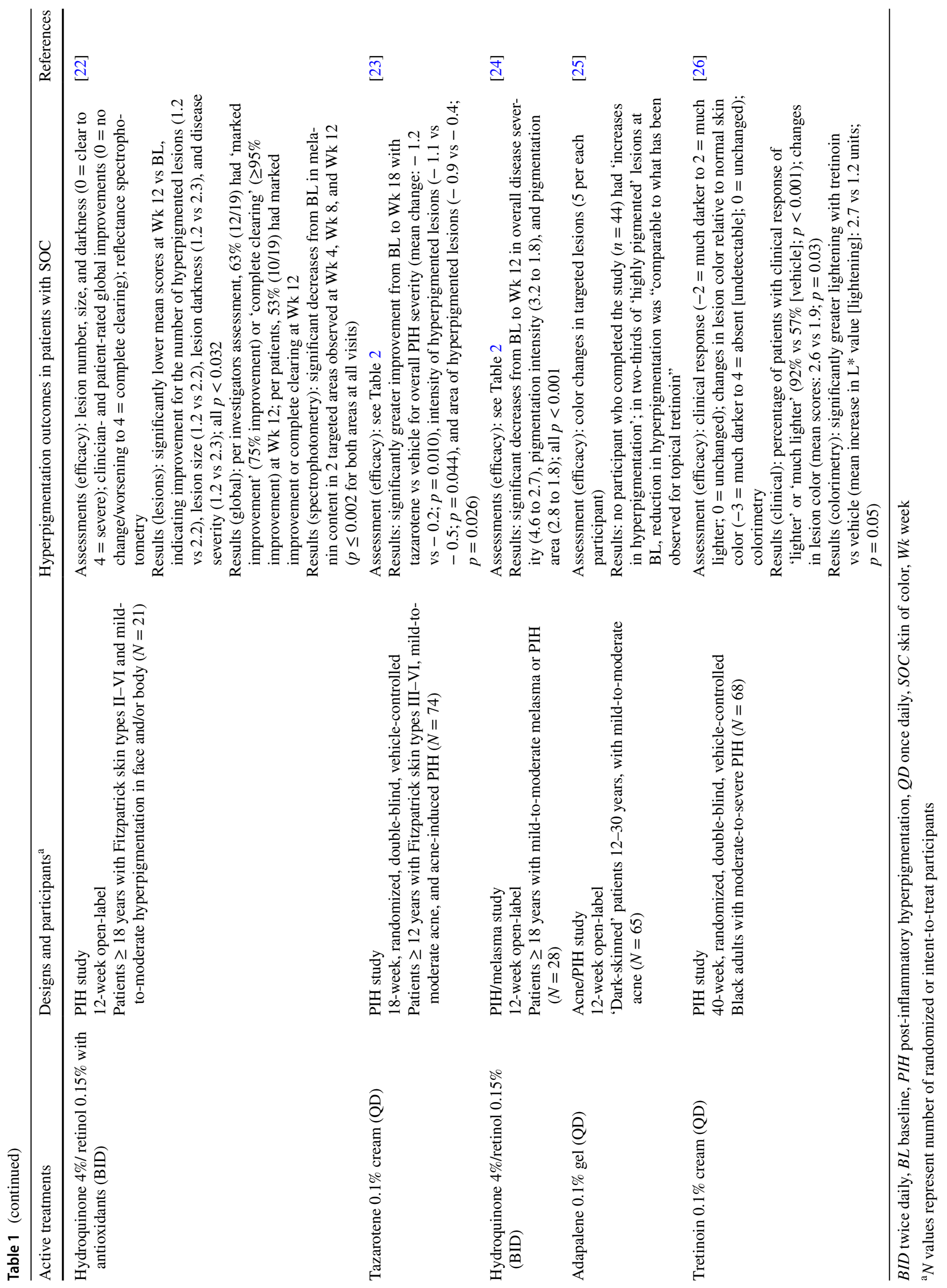


provide comparable efficacy to tazarotene cream with fewer side effects, as demonstrated in a phase II study of tazarotene $0.045 \%$ lotion which included tazarotene $0.1 \%$ cream as an active comparator [34].

The lotion formulations of tazarotene and tretinoin have been approved for facial acne, with pooled post-hoc analyses from the phase III trials showing statistically significant reductions in inflammatory and noninflammatory counts relative to vehicle after 12 weeks of treatment in participants with moderate-to-severe acne $[35,36]$. Although these retinoid lotions have not been specifically evaluated for hyperpigmentation, post-hoc analyses of safety results indicate improvements in hyperpigmentation among participants with SOC (Table 1).

In a post-hoc safety analysis of tretinoin $0.05 \%$ lotion that included 308 Black participants from the phase III studies, mean hyperpigmentation scores decreased from baseline to week 12 (from 0.8 to 0.6 ), as did the percentage of participants with moderate or severe hyperpigmentation (from 26.4 to $17.3 \%$ ) [17]. In a subgroup analysis that included 69 Asian study participants, hyperpigmentation was generally mild at baseline and remained mild throughout treatment (i.e., mean scores $<1$ at all visits) [18]. Among 766 Hispanic study participants, hyperpigmentation severity was progressively reduced with tretinoin $0.05 \%$ lotion. At baseline, $39 \%$ of participants had some degree of hyperpigmentation; at week $12,73 \%$ of participants had no hyperpigmentation [19].

In a post-hoc analysis that included 262 Black and 352 Hispanic participants, treatment with tazarotene $0.045 \%$ lotion decreased the percentage with any hyperpigmentation $(1=$ mild to $3=$ severe $)$ from baseline to week 12 as follows: Black, 40.5 to $31.3 \%$; Hispanic, 17.8 to $11.2 \%$ (Table 1) [15]. In a subgroup analysis that included 78 Asian participants, the percentage with any hyperpigmentation did not decrease; however, moderate hyperpigmentation decreased from $11.9 \%$ at baseline to $2.4 \%$ at week 12 [16]. Improvements in acne and hyperpigmentation after 12 weeks of treatment with tazarotene $0.045 \%$ lotion are illustrated in Figs. 2, 3 and 4.

Tazarotene $0.045 \%$ lotion might also be favorable for SOC patients in terms of potential irritability. Overall, pain, dryness, and exfoliation at the application site were the most common adverse events with tazarotene $0.045 \%$ lotion in the phase III studies [36, 37]. In all racial and ethnic subgroups, the incidence of these adverse events was 1-5\% except for pain, which was slightly higher in Black versus White participants (6.6\% vs 5.2\%) and Hispanic versus non-Hispanic participants (6.1\% vs $5.0 \%$ ) [15]. Exfoliation with tazarotene $0.045 \%$ lotion was highest in Black participants $(5.0 \%$ vs $2.5 \%$ [Hispanic] and $1.4 \%$ [White]), which might help to lighten PIH in melanin-rich skin. Notably, application-site irritation, dermatitis, and rash with tazarotene $0.045 \%$ lotion were reported in $\leq 1.2 \%$ of Hispanic participants, $\leq 0.8 \%$ of Black participants, and 0\% of Asian participants (Ortho Dermatologics, data on file).

Trifarotene $0.005 \%$ cream is a fourth-generation retinoid approved for the treatment of facial and truncal acne [38], but its clinical effects on PIH in patients with SOC have not yet been reported. However, results from animal models indicate that this medication may have some ability to reduce hyperpigmentation [39].

\section{Assessing Acne and Hyperpigmentation in Patients with Skin of Color (SOC)}

\subsection{Assessment Challenges}

Various global assessments are used to assess acne severity in clinical trials, including the Evaluator's Global Severity Score (EGSS), Investigator's Global Assessment (IGA), and Global Acne Assessment Score (GAAS), with ratings ranging from 'clear' to 'severe' or 'very severe' [40]. Similarly subjective scales have also been used to assess hyperpigmentation, such as an adaptation of IGA with scores/ratings ranging from $0=$ 'clear of hyperpigmentation' to $5=$ 'very severe hyperpigmentation (very dark brown, almost black in quality)' [41] and a cutaneous safety instrument (used in acne clinical trials) that includes a hyperpigmentation item with scores/ratings ranging from $0=$ 'none, no evidence' to $3=$ 'severe, marked/prominent' [37].

The challenge of assessing patients with SOC is that acne can be complicated by the presence of hyperpigmentation, and vice versa. For evaluating hyperpigmentation, a more multidimensional approach may be needed —one that addresses the intensity and distribution of hyperpigmented lesions as well as the potential unwanted effects of treatment (e.g., hypopigmentation and tolerability issues). One such example is the Post-inflammatory Hyperpigmentation Severity Scale (Table 2), which was used in the studies with clindamycin/tretinoin 1.2\%/0.025\% gel [20] and tazarotene $0.1 \%$ cream [23].

The challenge of evaluating hyperpigmentation in patients with SOC was illustrated in a study from the Asian Acne Board, which found that agreement about PIH frequency and severity can be quite low, even among clinical experts [42]. According to the authors, one major challenge in this study was differentiating PIH from erythema. This was complicated by the presence of active acne, which tended to cause more labeling of PIH as erythema, along with subjective differences among experts regarding lesional colors. Some experts considered PIH to include "lesions with a range of colors from light brown to purplish red" while others believed that "reddish lesions were properly identified as erythematous and not PIH." As the authors point out, PIH 

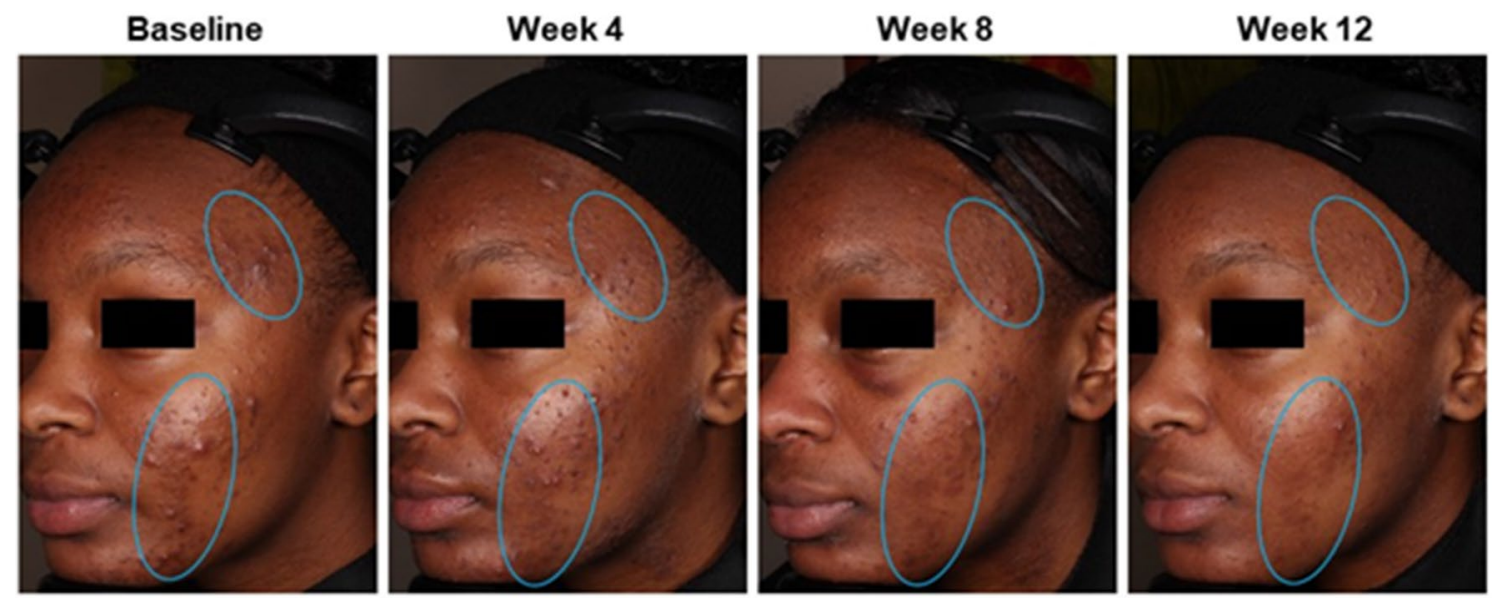

Fig. 2 Acne and hyperpigmentation improvements in a 15-year-old Black female patient who was treated with tazarotene $0.045 \%$ lotion once daily for 12 weeks. Clinical trial results: inflammatory lesion counts reduced by $80 \%$; noninflammatory lesion counts reduced by $33 \%$; EGSS scores at baseline $(4=$ severe $)$ and week $12(3=$ mod-

erate). Authors' assessments: Fitzpatrick skin type V; PIH Severity Scale grading at baseline $(4=$ moderate $)$ and week $12(2=$ mild $)$. Individual results may vary. EGSS Evaluator's Global Severity Score, $P I H$ post-inflammatory hyperpigmentation
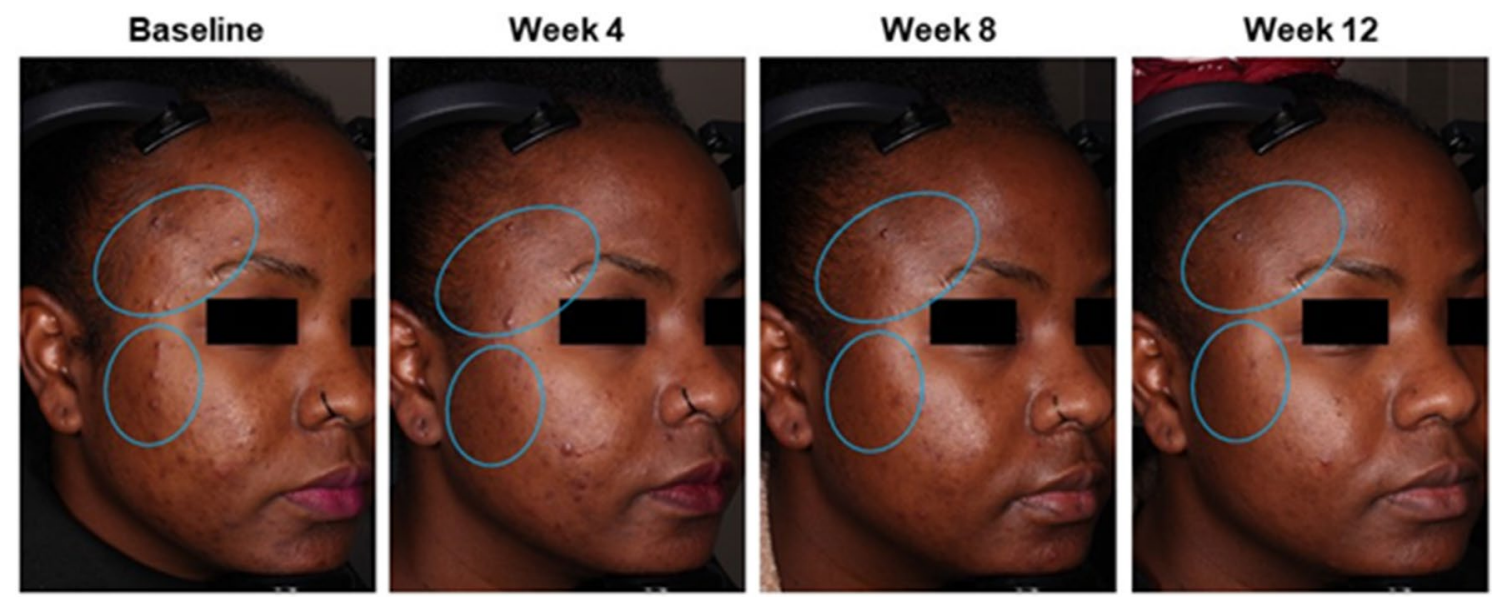

Fig. 3 Acne and hyperpigmentation improvements in a 27 -year-old Black female patient who was treated with tazarotene $0.045 \%$ lotion once daily for 12 weeks. Clinical trial results: inflammatory lesion counts reduced by $60 \%$; noninflammatory lesions counts reduced by $46 \%$; EGSS scores at baseline $(3=$ severe $)$ and week $12(2=$ mod-

has been described in the literature with "a range of colors from brown to reddish"-which does not offer sufficient guidance-and melanin-rich skin tones may make the visualization of both PIH and erythema more challenging.

Some of these assessment challenges may be addressed by clinician education and training, as was demonstrated in the Post-Acne Hyperpigmentation Index (PAHPI) validation study [43]. This study included six raters (two dermatology residents, two faculty dermatologists, and two pigmentary disorder specialists) who assessed acne-induced PIH in 21 adults with Fitzpatrick skin types III-VI. The PAHPI erate). Authors' assessments: Fitzpatrick skin type V; PIH Severity Scale grading at baseline $(4=$ moderate $)$ and week $12(0=$ normal $)$. Individual results may vary. EGSS Evaluator's Global Severity Score, $P I H$ post-inflammatory hyperpigmentation

was developed using feedback from patient focus groups, and weighted scores from the three domains of greatest patient concern-lesion size, lesion intensity, and number of lesions-were used to calculate the overall index score. Interrater reliability on PAHPI scores improved after raters completed a 30-min training program that included instructions for ignoring excoriations, inflammatory lesions, and other types of lesions. With training, both intra- and interrater reliability for total PAHPI scores were high, and the scores were well correlated to global disease severity, a melanin index, and a quality-of-life index. 

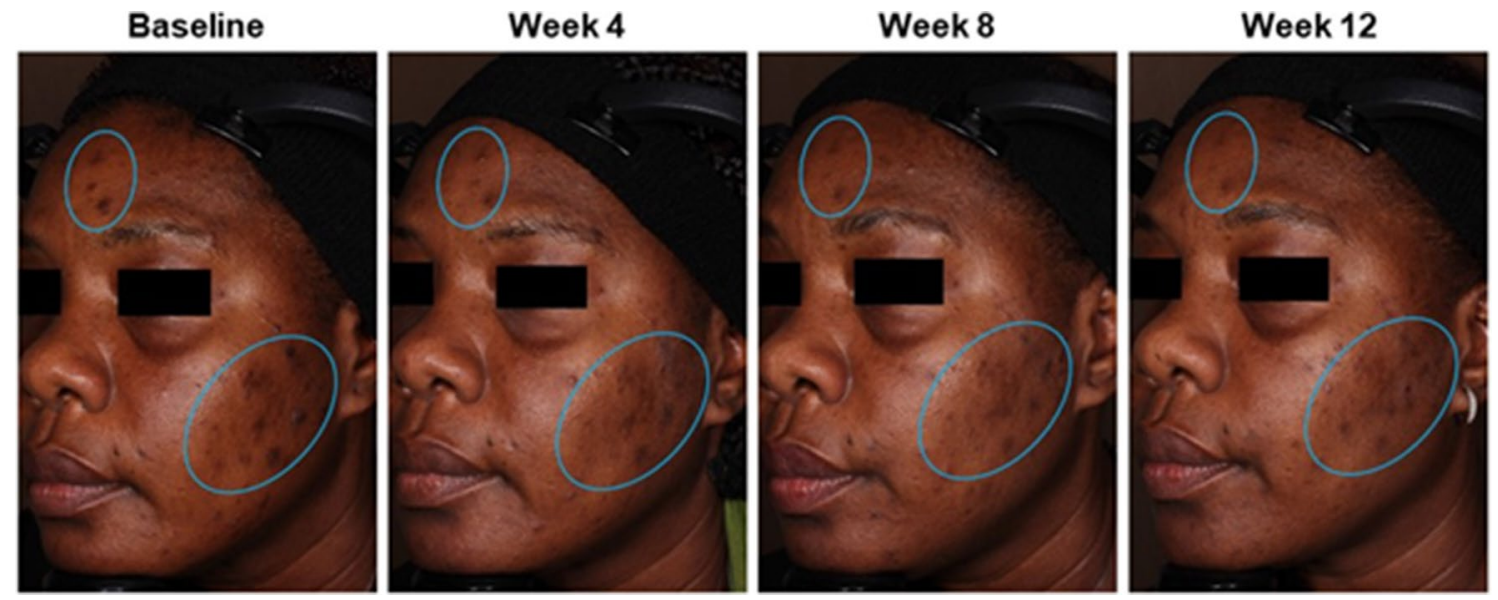

Fig. 4 Acne and hyperpigmentation improvements in a 50-year-old Black female patient who was treated with tazarotene $0.045 \%$ lotion once daily for 12 weeks. Clinical trial results: inflammatory lesion counts reduced by $90 \%$; noninflammatory lesions counts reduced by $79 \%$; EGSS scores at baseline $(3=$ severe $)$ and week $12(2=$ mod- erate). Authors' assessments: Fitzpatrick skin type VI; PIH Severity Scale grading at baseline $(7=$ marked/severe $)$ and week $12(4=$ moderate). Individual results may vary. EGSS Evaluator's Global Severity Score, $P I H$ post-inflammatory hyperpigmentation

Table 2 Post-inflammatory hyperpigmentation severity scale

\begin{tabular}{|c|c|c|c|c|c|}
\hline Grade & Overall disease severity & $\begin{array}{l}\text { Pigmentary intensity of } \\
\text { hyperpigmented lesions }\end{array}$ & $\begin{array}{l}\text { Area of hyperpigmented } \\
\text { lesions }\end{array}$ & $\begin{array}{l}\text { Degree of hypopigmenta- } \\
\text { tion }\end{array}$ & $\begin{array}{l}\text { Erythema, } \\
\text { burning, peel- } \\
\text { ing, dryness }\end{array}$ \\
\hline 0 & Normal & None (normal) & None & None & None \\
\hline 1 & Present but less than mild & Trace (mild and localized) & Trace $(1-10 \%$ of face $)$ & Trace (slight and localized) & Trace \\
\hline 2 & Mild (slightly noticeable) & Mild (mild and diffuse) & Mild (11-25\% of face) & Mild (slight and diffuse) & Mild \\
\hline 3 & Between mild and moderate & $\begin{array}{l}\text { Moderate (moderate and } \\
\text { diffuse) }\end{array}$ & Moderate (26- $40 \%$ of face) & $\begin{array}{l}\text { Moderate (noticeable and } \\
\text { diffuse) }\end{array}$ & Moderate \\
\hline 4 & Moderate (noticeable) & $\begin{array}{l}\text { Marked (moderate and } \\
\text { dense) }\end{array}$ & Marked (41-50\% of face) & $\begin{array}{l}\text { Marked (noticeable and } \\
\text { dense) }\end{array}$ & Marked \\
\hline 5 & $\begin{array}{l}\text { Between moderate and } \\
\text { marked }\end{array}$ & $\begin{array}{l}\text { Severe (prominent and } \\
\text { dense) }\end{array}$ & Severe $(>50 \%$ of face) & $\begin{array}{c}\text { Severe (complete lack of } \\
\text { melanin pigmentation) }\end{array}$ & Severe \\
\hline 6 & Marked (distinctive) & & & & \\
\hline 7 & Between marked and severe & & & & \\
\hline 8 & Severe (very distinctive) & & & & \\
\hline
\end{tabular}

Republished with permission from Callender VD et al, J Clin Aesthet Dermatol 2012 [20]; conveyed through Copyright Clearance Center, Inc.

Authors from the Asian Acne Board study called for the establishment of "objective and quantitative criteria" for the evaluation of PIH, while authors of the PAHPI validation study noted the importance of clinician training. Together, these conclusions point to the need for a more standardized approach to assessing PIH, which may be particularly important for investigating potential treatments. As shown in Table 1, various measures have been used to evaluate the effects of topical retinoids on PIH. Unless treatments are compared in headto-head trials, this variability makes it difficult to assess differences among therapies and identify which ones might be most appropriate for patients. Moving forward, standardization of assessments - coupled with investigator training-could contribute to a more unified body of research that addresses questions about the presentation, treatment, and resolution of $\mathrm{PIH}$ in patients of all skin types.

\subsection{Implications for Researchers and Clinicians}

\subsubsection{Clinical Trial Methods}

More standardization of methods across clinical trials can contribute to better assessment and treatment of acneinduced PIH in patients with SOC. Reporting Fitzpatrick skin types in clinical trials may be a good starting point. Although hyperpigmentation-focused studies have included 
Fitzpatrick skin types [22, 23], acne studies have tended to include race (per patient self-report) but not Fitzpatrick skin types. This can present difficulties for researchers who want to analyze study data in patients with SOC because patients within a race or ethnicity can have a range of skin tones.

Most contemporary clinical trials include lesion counts along with clinician-rated global assessments (e.g., IGA, EGSS) to assess acne severity. However, hyperpigmentation is either reported as a safety outcome (e.g., adverse event, cutaneous assessment) or not at all. Inclusion of a multidimensional hyperpigmentation scale as an efficacy outcome in acne studies - such as the one presented in Table 2-could help clinicians identify the presence and severity of hyperpigmentation in their patients with SOC and select appropriate treatments for those patients. In addition, clinical trials may need to be longer (e.g., 4- or 6-month duration) since hyperpigmentation tends to improve more slowly than acne. However, since PIH can improve over time without treatment, much longer trials (e.g., $\geq 6$ months) may make it difficult to distinguish between intrinsic improvements and treatment effects.

\subsubsection{Clinician Education}

Continued educational efforts are needed to address some of the key challenges of assessing hyperpigmentation in patients with SOC, as described below.

- Hyperpigmentation versus erythema. Acne sequelae include both hyperpigmentation and erythema. Hyperpigmentation (due to overproduction of melanin) is typically described as being brown or black in color, while erythema (due to capillary damage near the skin surface) is described as being red or purple. The perception of color differences vary across different skin tones, and distinguishing erythema from hyperpigmentation in higher phototypes may pose challenges for some clinicians.

- Subclinical inflammation and persistent hyperpigmentation. Conventional models of acne pathogenesis usually describe follicular hyperkeratinization (i.e., microcomedone formation) as the first step in the development of acne lesions, with eventual progression to visible inflammatory lesions [44]. However, a study using biopsied skin samples from acne and nonacne patients showed that inflammatory processes (e.g., increased expression of vascular adhesion molecule 1 , upregulation of interleukin-1) are likely involved in the very earliest stages of acne development, prior to the hyperproliferative changes that are seen in visible lesions [45]. This type of inflammation is described as 'subclinical' because it occurs before the appearance of visible acne lesions. Moreover, hyperpigmentation due (at least in part) to subclinical inflammation may be considered 'persistent' rather than post-inflammatory because the underlying inflammatory factors may still be present. Regardless of whether the mechanism is persistent or post-inflammatory, topical retinoids are likely to improve hyperpigmentation, whether administered alone or in combination with other treatments.

- Efficacy versus adverse effects. Some retinoid effects can be either beneficial or harmful, depending on each patient's particular circumstance (e.g., skin type sensitivity, history of scarring, treatment goals). For example, transient peeling and scaling are common after retinoid treatment is initiated. In some patients, these effects may be considered a desirable exfoliating property of the retinoid-one that helps make skin color more even. In others, these effects may be too drying or irritating, possibly causing unwanted post-inflammatory responses that make hyperpigmented lesions or patches worse than before treatment. Retinoids can also result in an overall lightening of the skin, which some patients want and others do not. Asking patients specific questions about their skin (e.g., normal, oily, dry, combination), skincare and haircare products (e.g., pomades, cosmetics, moisturizers), cleansing methods, and history of irritation with topical medications can help determine the best approach for retinoid treatment [46].

\section{Practical Approaches to Treating Acne and Hyperpigmentation in Patients with SOC}

\subsection{Patient Education}

Hyperpigmentation is a leading cause for dermatology visits among patients with SOC. Patients may find hyperpigmentation to be more bothersome than acne lesions [10], which can lead to frustration if hyperpigmentation does not improve with treatment-even if the acne is resolved. Therefore, it is important to educate patients about their dermatologic conditions and manage their expectations about treatment. Some points for discussion with patients are listed below.

- Acne-associated hyperpigmentation is due to inflammation from acne. It is important to treat the acne in order to prevent the development of more hyperpigmentation.

- Topical retinoids are approved for the treatment of acne and can be used as monotherapy or in combination with non-retinoid treatments [12]. They are effective in patients of all skin types and have also been shown to improve hyperpigmentation in patients with melanin- 
rich skin. These medications are ideal first-line therapies in patients with both acne and hyperpigmentation, unless contraindicated (e.g., during pregnancy).

- Retinoids will likely improve acne symptoms first, which is important because acne lesions and scarring can contribute to the development or worsening of hyperpigmentation.

- With continued retinoid use, improvements in hyperpigmentation can be expected. However, it is important to apply retinoids as prescribed (to avoid more irritation), practice good skin hygiene, and use sunscreen.

- Treatment regimens may change over time, based on the patient's response to medication(s) and tolerability. Ongoing communication between patients and clinicians about symptom improvement and side effects is strongly encouraged.

\subsection{Irritation and Skin Care}

The goal of treatment is to maximize efficacy while minimizing irritation and other side effects. Irritation can exacerbate hyperpigmentation in patients with SOC, but measures can be taken to offset this risk. First, newer retinoid formulations (e.g., lower-concentration lotions for tretinoin and tazarotene) may be less irritating than older gel or cream formulations, while offering the same level of efficacy. These newer formulations counter the misconception that irritation is a sign that the retinoid is working. Second, patients should avoid toners and astringents that can overly dry the skin and increase irritation. Patients should also avoid haircare and cosmetic products that contain comedogenic ingredients. Finally, use of a $\mathrm{pH}$-balanced cleanser and non-comedogenic moisturizer can be helpful. Many dermatologists have been trained to recommend that moisturizers be applied after retinoids. However, results from a percutaneous penetration study of azelaic acid using human skin samples [47] and a clinical trial of clindamycin/phosphate and tretinoin $0.05 \%$ gel in acne patients [48] suggest that moisturizers that increase hydration and improve barrier function may be applied before topical treatment without affecting absorption. For patients with sensitive skin, applying moisturizer before the retinoid may help reduce irritation.

\subsection{Early Treatment and Long-Term Management}

Early and effective treatment of acne is key to reducing long-term sequelae such as hyperpigmentation and scarring (including keloids), which can be very challenging to treat in patients with SOC. Topical retinoids have anti-inflammatory as well as comedolytic effects, which make them ideal for treating both acne and hyperpigmentation. They should therefore be considered a first-line therapy for most patients, unless contraindicated or not well tolerated. For patients who experience cutaneous reactions to topical retinoids, trial with a newer formulation may be warranted.

Retinoids are an essential part of treatment, but many patients may require a multimodal approach to address the different disease mechanisms associated with acne and hyperpigmentation. Topical treatments that may be used in conjunction with topical retinoids include the following:

- Azelaic acid. This naturally occurring acid has antiinflammatory, anti-infective, and anti-oxidant properties [49]. It may reduce hyperpigmentation by inhibiting tyrosinase, which is essential for melanin production [50]. Studies with azelaic acid $15 \%$ foam have demonstrated improvements in acne [51, 52]; a study with azelaic acid $15 \%$ gel showed improvements in hyperpigmentation [53].

- Antibiotics with benzoyl peroxide (BP). Combining topical antibiotics (e.g., clindamycin, erythromycin) with BP reduces the risk for resistance. In addition to their proven effects on acne [12], these combination therapies may be effective in reducing hyperpigmentation, as demonstrated with clindamycin/BP $1 \% / 5 \%$ gel in patients with SOC [54].

- Dapsone. Dapsone has both anti-microbial and antiinflammatory properties, which allow for its use in a wide range of dermatologic conditions [55]. Dapsone $7.5 \%$ gel is approved for the treatment of acne, and a post-hoc analysis of the phase III trials showed no significant differences in efficacy between patients with SOC (defined as Fitzpatrick skin type IV-VI) and those with lower skin phototype (type I-III) [56]. No results for hyperpigmentation were presented, but patterns of investigator-rated tolerability (i.e., stinging/burning, dryness, scaling, erythema) were similar between subgroups.

- Hydroquinone. Hydroquinone inhibits melanin production by acting as an alternative substrate for tyrosinase, with possible destruction of melanocytes and degradation of melanosomes [4]. Hydroquinone has been a mainstay for skin lightening, and its effects may be further enhanced by combination with a topical retinoid [57]. The triple combination of fluocinolone acetonide/hydroquinone/tretinoin $(0.01 \% / 4 \% / 0.05 \%$ cream $)$ is approved for short-term treatment of moderate-to-severe melasma in the face. For acne-induced PIH, patients may need to carefully apply topical hydroquinone to affected areas to avoid unwanted hypopigmentation.

Some patients with SOC may require oral antibiotics as part of their acne regimen, and strategies such as an initial oral antibiotic (e.g., doxycycline, minocycline) plus topical retinoid followed by long-term retinoid maintenance 
can be beneficial [30]. Oral isotretinoin may be considered in patients with severe inflammatory acne who are at risk for scarring [30]. Spironolactone, an antiandrogen that prevents sebum production, may be beneficial in patients with hormonal acne [58]. Procedural therapies such superficial chemical peels and non-ablative fractional laser therapy can also be used in conjunction with topical treatments to reduce hyperpigmentation [4]. Caution should be taken with more invasive procedures, such as medium- or deep-skin peels [2]. Retinoid treatment can be temporarily stopped before chemical peels to reduce the risk of crusting and skin erosion [2].

\section{Conclusions}

For patients with SOC, hyperpigmentation is one of the most prevalent and bothersome dermatologic conditions. Since acne is a common cause of hyperpigmentation, early and aggressive treatment of acne is essential. Topical retinoids are highly effective in treating acne and can also improve hyperpigmentation due to their anti-inflammatory properties. For patients with sensitive skin, the newer lotions may be less irritating than older retinoid formulations. Educating patients about how acne treatments work and how long they take to work (for both acne and hyperpigmentation)—along with clear directions for skin care (cleansing, moisturizing, and sunscreen)—can help manage their expectations and improve treatment outcomes.

Acknowledgements Medical writing support was provided by Mildred Bahn (Prescott Medical Communications Group, Chicago, IL, USA) with financial support from Ortho Dermatologics. Ortho Dermatologics is a division of Bausch Health US, LLC.

\section{Declarations}

Funding Medical writing support for this review article was funded by Ortho Dermatologics. Ortho Dermatologics is a division of Bausch Health US, LLC.

Conflicts of interest Valerie Callender has served as an investigator, consultant, or speaker for AbbVie, Beiersdorf, Eli Lilly, Galderma, InCyte, L'Oréal, Ortho Dermatologics, Pfizer, Revance, Sente Labs, $\mathrm{UCB}$ and Vyne. Hilary Baldwin has served as advisor, investigator, and on speakers' bureaus for Almirall, Cassiopea, Vyne, Galderma, Ortho Dermatologics, Sol Gel, and Sun Pharma. Fran Cook-Bolden has served as consultant, speaker, investigator for Galderma, LEO Pharma, Almirall, Cassiopea, Ortho Dermatologics, Investigators Encore, Foamix, Hovione, Aclaris, Cutanea. Andrew Alexis has received grant/research support (funds to institution) from Leo, Novartis, Almirall, Bristol-Myers-Squibb, Amgen, Menlo, Galderma, Valeant (Bausch Health), Cara, Arcutis; has served as a consultant/advisory board member for Leo, Novartis, Menlo, Galderma, Pfizer, SanofiRegeneron, Dermavant, Unilever, Beiersdorf, Valeant, L'Oreal, BMS, Menlo, Scientis, Bausch Health, UCB, Vyne, Cassiopea, Arcutis, Janssen, Allergan, Almirall, Abbvie, Sol-Gel, Amgen; and has served as a speaker for Pfizer, Sanofi-Genzyme/Regeneron, Astra Zeneca. Linda
Stein Gold has served as an investigator/consultant or speaker for Ortho Dermatologics, LEO Pharma, Dermavant, Incyte, Novartis, AbbVie, Pfizer, Sun Pharma, UCB, Arcutis, Vyne, Sol-Gel, and Lilly. Eric Guenin is an employee of Ortho Dermatologics and may hold stock and/or stock options in its parent company.

Ethics approval Not applicable.

Consent to participate Not applicable.

Code availability Not applicable.

Consent for publication Patients provided written informed consent for photographs to be used for educational purposes.

Availability of data Not applicable.

Author contributions All authors made substantial contributions to the conception and design of the work; drafted the work/revised it critically; approved the version to be published; and agree to be accountable for all aspects of the work

Open Access This article is licensed under a Creative Commons Attribution-NonCommercial 4.0 International License, which permits any non-commercial use, sharing, adaptation, distribution and reproduction in any medium or format, as long as you give appropriate credit to the original author(s) and the source, provide a link to the Creative Commons licence, and indicate if changes were made. The images or other third party material in this article are included in the article's Creative Commons licence, unless indicated otherwise in a credit line to the material. If material is not included in the article's Creative Commons licence and your intended use is not permitted by statutory regulation or exceeds the permitted use, you will need to obtain permission directly from the copyright holder. To view a copy of this licence, visit $\mathrm{http} / / /$ creativecommons.org/licenses/by-nc/4.0/.

\section{References}

1. Yin NC, McMichael AJ. Acne in patients with skin of color: practical management. Am J Clin Dermatol. 2014;15(1):7-16.

2. Alexis AF, Harper JC, Stein Gold LF, Tan JKL. Treating acne in patients with skin of color. Semin Cutan Med Surg. 2018;37(3s):S71-3.

3. Lawrence E, Al Aboud KM. Postinflammatory hyperpigmentation. Treasure Island: StatPearls; 2020.

4. Kaufman BP, Aman T, Alexis AF. Postinflammatory hyperpigmentation: epidemiology, clinical presentation, pathogenesis and treatment. Am J Clin Dermatol. 2018;19(4):489-503.

5. Davis EC, Callender VD. Postinflammatory hyperpigmentation: a review of the epidemiology, clinical features, and treatment options in skin of color. J Clin Aesthet Dermatol. 2010;3(7):20-31.

6. Taylor SC, Cook-Bolden F, Rahman Z, Strachan D. Acne vulgaris in skin of color. J Am Acad Dermatol. 2002;46(2)(Suppl 2):S98-106.

7. Chua-Ty G, Goh CL, Koh SL. Pattern of skin diseases at the National Skin Centre (Singapore) from 1989-1990. Int J Dermatol. 1992;31(8):555-9.

8. Perkins AC, Cheng CE, Hillebrand GG, Miyamoto K, Kimball AB. Comparison of the epidemiology of acne vulgaris among Caucasian, Asian, Continental Indian and African American women. J Eur Acad Dermatol Venereol. 2011;25(9):1054-60. 
9. Gorelick J, Daniels SR, Kawata AK, Degboe A, Wilcox TK, Burk CT, et al. Acne-related quality of life among female adults of different races/ethnicities. J Dermatol Nurses Assoc. 2015;7(3):154-62.

10. Callender VD, Alexis AF, Daniels SR, Kawata AK, Burk CT, Wilcox TK, et al. Racial differences in clinical characteristics, perceptions and behaviors, and psychosocial impact of adult female acne. J Clin Aesthet Dermatol. 2014;7(7):19-31.

11. Alexis AF, Woolery-Lloyd H, Williams K, Andriessen A, Callender VD, Kang S, et al. Racial/ethnic variations in acne: implications for treatment and skin care recommendations for acne patients with skin of color. J Drugs Dermatol. 2021;20(7):716-25.

12. Zaenglein AL, Pathy AL, Schlosser BJ, Alikhan A, Baldwin HE, Berson DS, et al. Guidelines of care for the management of acne vulgaris. J Am Acad Dermatol. 2016;74(5):945-73 e33.

13. Chien A. Retinoids in acne management: review of current understanding, future considerations, and focus on topical treatments. J Drugs Dermatol. 2018;17(12):s51-5.

14. Leyden J, Stein-Gold L, Weiss J. Why topical retinoids are mainstay of therapy for acne. Dermatol Ther (Heidelb). 2017;7(3):293-304.

15. Bhatia N, Weiss JS, Sadick N, Cook-Bolden FE, Tyring SK, Guenin E, et al. Novel polymeric tazarotene $0.045 \%$ lotion for moderate-to-severe acne: pooled phase 3 analysis by race/ethnicity. J Drugs Dermatol. 2020;19(7):727-34.

16. Cook-Bolden FE, Bhatia N, Alexis AF, Green L, Stein Gold L, Han G, et al. Polymeric emulsion tazarotene $0.045 \%$ lotion for the once-daily treatment of moderate-to-severe acne vulgaris in Asian participants [poster]. In: Presented virtually at the Skin of Color Society annual scientific symposium, August 31, 2021.

17. Bhatia ND, Werschler WP, Cook-Bolden FE, Guenin E. Tolerability of tretinoin lotion $0.05 \%$ for moderate to severe acne vulgaris: a post hoc analysis in a black population. Cutis. 2020;106(1):45-50.

18. Han G, Armstrong AW, Desai SR, Guenin E. Novel tretinoin $0.05 \%$ lotion for the once-daily treatment of moderate-tosevere acne vulgaris in an Asian population. J Drugs Dermatol. 2019;18(9):910-6.

19. Cook-Bolden FE, Weinkle SH, Guenin E, Bhatt V. Novel tretinoin $0.05 \%$ lotion for once-daily treatment of moderate-to-severe acne vulgaris in a Hispanic population. J Drugs Dermatol. 2019;18(1):32-8.

20. Callender VD, Young CM, Kindred C, Taylor SC. Efficacy and safety of clindamycin phosphate $1.2 \%$ and tretinoin $0.025 \%$ gel for the treatment of acne and acne-induced post-inflammatory hyperpigmentation in patients with skin of color. J Clin Aesthet Dermatol. 2012;5(7):25-32.

21. Tanghetti E, Dhawan S, Green L, Del Rosso J, Draelos Z, Leyden $\mathrm{J}$, et al. Randomized comparison of the safety and efficacy of tazarotene $0.1 \%$ cream and adapalene $0.3 \%$ gel in the treatment of patients with at least moderate facial acne vulgaris. J Drugs Dermatol. 2010;9(5):549-58.

22. Cook-Bolden FE, Hamilton SF. An open-label study of the efficacy and tolerability of microencapsulated hydroquinone $4 \%$ and retinol $0.15 \%$ with antioxidants for the treatment of hyperpigmentation. Cutis. 2008;81(4):365-71.

23. Grimes P, Callender V. Tazarotene cream for postinflammatory hyperpigmentation and acne vulgaris in darker skin: a double-blind, randomized, vehicle-controlled study. Cutis. 2006;77(1):45-50.

24. Grimes PE. A microsponge formulation of hydroquinone $4 \%$ and retinol $0.15 \%$ in the treatment of melasma and postinflammatory hyperpigmentation. Cutis. 2004;74(6):362-8.

25. Jacyk WK. Adapalene in the treatment of African patients. J Eur Acad Dermatol Venereol. 2001;15(Suppl 3):37-42.

26. Bulengo-Ransby SM, Griffiths CE, Kimbrough-Green CK, Finkel LJ, Hamilton TA, Ellis CN, et al. Topical tretinoin (retinoic acid) therapy for hyperpigmented lesions caused by inflammation of the skin in black patients. N Engl J Med. 1993;328(20):1438-43.

27. Chandraratna RA. Tazarotene: the first receptor-selective topical retinoid for the treatment of psoriasis. J Am Acad Dermatol. 1997;37(2 Pt 3):S12-7.

28. Wang CQF, Akalu YT, Suarez-Farinas M, Gonzalez J, Mitsui $\mathrm{H}$, Lowes MA, et al. IL-17 and TNF synergistically modulate cytokine expression while suppressing melanogenesis: potential relevance to psoriasis. J Investig Dermatol. 2013; 133(12):2741-52.

29. Avage (tazrotene $0.1 \%$ cream). Prescribing information. Madison NJ: Allergan USA, Dec 2019.

30. Alexis AF. Acne vulgaris in skin of color: understanding nuances and optimizing treatment outcomes. J Drugs Dermatol. 2014;13(6):s61-5.

31. Baldwin H, Webster G, Stein Gold L, Callender V, Cook-Bolden FE, Guenin E. 50 years of topical retinoids for acne: evolution of treatment. Am J Clin Dermatol. 2021;22(3):315-27.

32. Kircik LH, Draelos ZD, Berson DS. Polymeric emulsion technology applied to tretinoin. J Drugs Dermatol. 2019;18(4):s148-54.

33. Tanghetti EA, Stein Gold L, Del Rosso JQ, Lin T, Angel A, Pillai R. Optimized formulation for topical application of a fixed combination halobetasol/tazarotene lotion using polymeric emulsion technology. J Dermatol Treat. 2021;32(4):391-8.

34. Tanghetti EA, Kircik LH, Green LJ, Guenin E, Harris S, Martin G, et al. A phase 2, multicenter, double-blind, randomized, vehiclecontrolled clinical study to compare the safety and efficacy of a novel tazarotene $0.045 \%$ lotion and tazarotene $0.1 \%$ cream in the treatment of moderate-to-severe acne vulgaris. J Drugs Dermatol. 2019;18(6):542.

35. Tyring SK, Kircik LH, Pariser DM, Guenin E, Bhatt V, Pillai R. Novel tretinoin $0.05 \%$ lotion for the once-daily treatment of moderate-to-severe acne vulgaris: assessment of efficacy and safety in patients aged 9 years and older. J Drugs Dermatol. 2018;17(10):1084-91.

36. Tanghetti EA, Werschler WP, Lain E, Guenin E, Harris S, Loncaric A, et al. Novel polymeric lotion formulation of once-daily tazarotene $(0.045 \%)$ for moderate-to-severe acne: pooled phase 3 analysis. J Drugs Dermatol. 2020;19(3):272-9.

37. Tanghetti EA, Werschler WP, Lain T, Guenin E, Martin G, Pillai R. Tazarotene $0.045 \%$ lotion for once-daily treatment of moderateto-severe acne vulgaris: results from two phase 3 trials. J Drugs Dermatol. 2020;19(1):70-7.

38. Bell KA, Brumfiel CM, Haidari W, Boger L. Trifarotene for the treatment of facial and truncal acne. Ann Pharmacother. 2021;55(1):111-6.

39. Aubert J, Piwnica D, Bertino B, Blanchet-Rethore S, Carlavan I, Deret S, et al. Nonclinical and human pharmacology of the potent and selective topical retinoic acid receptor-gamma agonist trifarotene. Br J Dermatol. 2018;179(2):442-56.

40. Zarchi K, Jemec GBE. Severity assessment and outcome measures in acne vulgaris. Curr Derm Rep. 2012;1:131-6.

41. Isedeh P, Kohli I, Al-Jamal M, Agbai ON, Chaffins M, Devpura S, et al. An in vivo model for postinflammatory hyperpigmentation: an analysis of histological, spectroscopic, colorimetric and clinical traits. Br J Dermatol. 2016;174(4):862-8.

42. Goh CL, Abad-Casintahan F, Chow SK, Kubba R, Miyachi Y, Noppakun N, et al. Evaluating acne-related post-inflammatory hyperpigmentation is a challenge even amongst experts. J Dermatol. 2014;41(12):1106-8.

43. Savory SA, Agim NG, Mao R, Peter S, Wang C, Maldonado $\mathrm{G}$, et al. Reliability assessment and validation of the postacne hyperpigmentation index (PAHPI), a new instrument to measure postinflammatory hyperpigmentation from acne vulgaris. J Am Acad Dermatol. 2014;70(1):108-14. 
44. Del Rosso JQ, Kircik LH. The sequence of inflammation, relevant biomarkers, and the pathogenesis of acne vulgaris: what does recent research show and what does it mean to the clinician? J Drugs Dermatol. 2013;12(8 Suppl):s109-15.

45. Jeremy AH, Holland DB, Roberts SG, Thomson KF, Cunliffe WJ. Inflammatory events are involved in acne lesion initiation. J Investig Dermatol. 2003;121(1):20-7.

46. Davis EC, Callender VD. A review of acne in ethnic skin: pathogenesis, clinical manifestations, and management strategies. J Clin Aesthet Dermatol. 2010;3(4):24-38.

47. Del Rosso JQ, Lehman PA, Raney SG. Impact of order of application of moisturizers on percutaneous absorption kinetics: evaluation of sequential application of moisturizer lotions and azelaic acid gel 15\% using a human skin model. Cutis. 2009;83(3):119-24.

48. Zeichner JA, Patel RV, Haddican M, Wong V. Efficacy and safety of a ceramide containing moisturizer followed by fixed-dose clindamycin phosphate $1.2 \%$ /benzoyl peroxide $2.5 \%$ gel in the morning in combination with a ceramide containing moisturizer followed by tretinoin $0.05 \%$ gel in the evening for the treatment of facial acne vulgaris. J Drugs Dermatol. 2012;11(6):748-52.

49. Searle T, Ali FR, Al-Niaimi F. The versatility of azelaic acid in dermatology. J Dermatol Treat. 2020:1-11. https://doi.org/10. 1080/09546634.2020.1800579.

50. Schulte BC, Wu W, Rosen T. Azelaic acid: evidence-based update on mechanism of action and clinical application. J Drugs Dermatol. 2015;14(9):964-8.
51. Hoffman LK, Del Rosso JQ, Kircik LH. The efficacy and safety of azelaic acid $15 \%$ foam in the treatment of truncal acne vulgaris. J Drugs Dermatol. 2017;16(6):534-8.

52. Hashim PW, Chen T, Harper JC, Kircik LH. The efficacy and safety of azelaic acid $15 \%$ foam in the treatment of facial acne vulgaris. J Drugs Dermatol. 2018;17(6):641-5.

53. Kircik LH. Efficacy and safety of azelaic acid (AzA) gel 15\% in the treatment of post-inflammatory hyperpigmentation and acne: a 16-week, baseline-controlled study. J Drugs Dermatol. 2011;10(6):586-90.

54. Tanghetti E. Fixed-combination clindamycin 1\%-benzoyl peroxide 5\% hydrating gel: a flexible component of acne management. Cutis. 2009;84(5 Suppl):18-24.

55. Ghaoui N, Hanna E, Abbas O, Kibbi AG, Kurban M. Update on the use of dapsone in dermatology. Int J Dermatol. 2020;59(7):787-95.

56. Taylor SC, Cook-Bolden FE, McMichael A, Downie JB, Rodriguez DA, Alexis AF, et al. Efficacy, safety, and tolerability of topical dapsone gel, $7.5 \%$ for treatment of acne vulgaris by fitzpatrick skin phototype. J Drugs Dermatol. 2018;17(2):160-7.

57. Huerth KA, Hassan S, Callender VD. Therapeutic insights in melasma and hyperpigmentation management. J Drugs Dermatol. 2019;18(8):718-29.

58. Han JJ, Faletsky A, Barbieri JS, Mostaghimi A. New acne therapies and updates on use of spironolactone and isotretinoin: a narrative review. Dermatol Ther. 2021;11(1):79-91. 